\title{
Radial-axial transport coordination enhances sugar translocation in the phloem vasculature of plants
}

\author{
Mazen Nakad $^{1}$, Jean-Christophe Domec ${ }^{1,2}$, Sanna Sevanto ${ }^{3}$, Gabriel Katul ${ }^{1,4}$ \\ ${ }^{1}$ Nicholas School of the Environment, Duke University, Durham, NC, USA \\ ${ }^{2}$ Bordeaux Sciences Agro, UMR 1391 INRA-ISPA, France \\ ${ }^{3}$ Earth and Environmental Sciences Division, Los Alamos National Laboratory, Los Alamos, NM, USA \\ ${ }^{4}$ Department of Civil and Environmental Engineering, Duke University, Durham, NC, USA
}

Summary: The overall speed of sap increased by including a concentrationdependent viscosity in axial and radial directions.

Author contributions: G.K conceived the original screening and research plans, JC.D., S.S. and G.K supervised the progress of the model and numerical analysis, M.N conceived the project and wrote the article with contributions of all the authors, M.N. agrees to serve as the author responsible for contact and ensures communication.

Funding: This work was supported by the U.S. National Science Foundation and Los Alamos Directed Research and Development Exploratory Research Grant.

Corresponding author: Mazen Nakad, mazen.nakad@duke.edu 


\section{Abstract}

Understanding the controls of mass transport of photosynthates in the phloem of plants is necessary for describing plant carbon allocation, productivity, and responses to water and thermal stress. While several hypotheses about optimization of phloem structure and function, and limitations of phloem transport under drought have been tested both with models and anatomical data, the true impact of radial water exchange of phloem conduits with their surroundings on mass transport of photosynthates has not been addressed. Here the physics of the Münch mechanism of sugar transport is re-evaluated to include local variations in viscosity resulting from the radial water exchange in two dimensions (axial and radial). Model results show that radial water exchange pushes sucrose away from conduit walls thereby reducing wall frictional stress due to a decrease in sap viscosity and increasing sugar concentration in the central region of the conduit. This leads to increased sugar front speed and axial mass transport for a wide range of phloem conduit lengths and allows sugar transport to operate more efficiently than predicted by previous models. A faster front speed leads to higher phloem resiliency under drought because more sugar can be transported with a smaller pressure gradient.

\section{Introduction}

The efficiency of photosynthate transport from the production sites (sources; usually leaves) to areas of consumption or storage (sinks) within the vascular tissue known as the phloem is drawing significant attention in plant physiology. The implications of efficient photosynthate transport range from local impacts on tissue or plant health and growth to ecosystem-scale effects on carbon and water cycling because of the potential link between phloem transport and stomatal control of photosynthesis (Nikinmaa et al. 2013), and a possible link between phloem transport failure and plant mortality under drought (Sevanto et al. 2014). Consequently, several models for phloem transport and the connection between phloem structure and function, as well as for the potential weak points in the transport system have been formulated (Münch 1930, Phillips \& Dungan 1993, Thompson \& Holbrook 2003, Jensen et al. 2009, 2012, Sevanto 2014). The most commonly accepted concept under which all these models operate is that phloem vasculature is optimized for efficient transport of soluble organic compounds (mostly sugars) produced during photosynthesis approximately as described by the pressure-flow hypothesis or Münch mechanism (Münch 1930). In the pressure-flow hypothesis, transport 
is initiated in leaves when sugars and other metabolic products are loaded into the phloem. Once in the phloem, sugars and water molecules are driven to move through the phloem's complex network of narrow but elongated, interconnected, cylindrical living cells (sieve tubes) spanning the length of the plant following pressure gradients. High sugar concentration at the loading site (leaves) draws water from the xylem, the tissue supplying water to the leaves or other surrounding tissues by osmosis towards the phloem. At the sinks, sugars are unloaded from the phloem to growing or storage cells, and water is released back to the xylem or other surrounding tissues. The loading and unloading at sources and sinks build a pressure gradient in the phloem creating a system where plant water and photosynthate transport over long distances occurs without active pumping. This cycle of pressure buildup and transport without active pumping endowed the phloem system with the label miracle of ingenuity (van Bel 2003).

The simplicity, plausibility, and intuitive appeal of the Münch mechanism lead to its proliferation in mathematical models (Nikinmaa et al. 2013, Jensen et al. 2016). It is routinely used to connect plant carbon sources and sinks, and their concomitant controls in a future $\mathrm{CO}_{2}$ enriched climate (Mencuccini \& Hölttä 2010, Fatichi et al. 2019), and it has been used to explain aspects of plant hydraulic failure during drought (Savage et al. 2017, Konrad et al. 2018, Huang et al. 2018, Sevanto 2018, Salmon et al. 2019) and extreme cold temperatures (Swanson \& Geiger 1967, Wardlaw 1968). The direct consequence of those two abiotic stresses should be a decrease in the overall phloem flow rate because the viscosity of a sucrose solution increases significantly with the drought-induced increase in sugar concentration required for osmoregulation (Hölttä et al. 2009) and with decreasing temperature.

However, the Münch mechanism is not free from controversy. The main critique stems from the fact that the sieve tubes seem to have too low of a hydraulic conductance along the phloem to allow sugars to be transported from leaves to roots in the largest and longest of plants (Curtis \& Scofield 1933, Lang 1979, Fensom 1981, Knoblauch et al. 2016, Liesche \& Schulz 2018). These studies also report lower leaf sucrose concentration in tall trees compared to shorter vegetation. When interpreted using simplified transport models for hydraulic conductance, this suggests that the Münch mechanism cannot produce effective transport in tall plants because the driving force for sucrose transport is lower for a longer path length. To resolve the issues of inadequate conductance and too low-pressure gradient to efficiently drive flow in tall plants, it has been suggested 
that rather than exchanging water and sugars only at the extreme source and sink ends of the phloem pathway, as suggested by the original Münch mechanism, sugars and water could be exchanged at different locations along the pathway essentially forming a "relay" system to facilitate transport (Lang 1979). While plausible, and based on modeling studies, also effective in increasing transport capacity (Hölttä et al. 2009), there currently is no clear evidence for unloading and reloading of sugars from and to phloem conduits along the transport pathway. There is, however, an increasing amount of evidence that water might readily be exchanged between the conduits and their surroundings along the entire length of the pathway (Knoblauch \& Oparka 2012, Knoblauch \& Peters 2017, Stanfield et al. 2017).

Answering the question of whether and how easily phloem conduits exchange water with their surroundings outside the primary loading and unloading zones at sources and sinks is becoming necessary for evaluating the validity of the Münch mechanism, and because it determines how phloem transport is affected under stress (Sevanto 2014, 2018). Theoretically, if no water exchange occurs, plants run a risk of blocking phloem flow by viscosity increase and reduced pressure gradient under drought conditions because higher amounts of sugar are needed in the phloem conduits at the loading and unloading zones for osmoregulation against the declining water potential of the xylem and the surrounding tissues. If water exchange occurs readily along the entire transport pathway, the flow may not be restricted by the same constraints that stem from the original interpretation of the Münch mechanism (Phillips \& Dungan 1993, Sevanto 2014, Sevanto et al. 2014, Sevanto 2018). In particular, the effects of viscosity buildup can be ameliorated because of the diluting effects of radial water flow velocity, the focus of the work here.

Experimental challenges in measuring water and solute fluxes within the phloem (Curtis \& Scofield 1933, Housley \& Fisher 1977) has led to reliance on mathematical models of simplified phloem transport to understand transport mechanisms in the phloem. As expected when employing such models, values of one or more variables may not be well constrained or are uncertain, possibly by several orders of magnitude. This fact is often used to justify (overly) simplified description of transport physics in models. These simplifications might lead to biased mass fluxes and estimates for total transport. An alternative to the simplification approach is to assess the effects of the simplification on the results, and in the case of phloem transport, test whether the pressure-flow hypothesis predicts increases or decreases in sugar mass flux when these simplifications are re- 
laxed or re-addressed. If increases in mass flux can be demonstrated upon re-addressing key simplifications used with the Münch mechanism, the contradictions with some observations might be explained by the effects of these model simplifications lending further support to the Münch mechanism.

Irrespective of the physics of phloem transport, phloem anatomical structure is assumed to have evolved to optimize sugar transport. Within the confines of the Münch mechanism, this optimization arises because of trade-offs between benefits of increasing sugar concentration $(c)$ and its impact on the mass flux $J$. Increasing $c$ increases flux $(J)$ because the osmotic pressure driving water movement increases approximately linearly with $c$ (Van't Hopf equation); however, increasing $c$ is accompanied by a nonlinear increase in dynamic (and kinematic) viscosity thereby enhancing the viscous forces that oppose movement (drag) thereby reducing $J$. In most phloem transport models today, viscosity is treated either as a constant or it is allowed to vary with loaded sugar concentration assuming that radial water flow does not significantly affect sugar concentration or viscosity. Theoretical representation of $J$ along with a number of scaling arguments results in a maximal sugar flux $J_{\max }$ at around $c=20 \%$ (Jensen et al. 2013) independent of the sieve tube geometric properties. Interestingly, upon averaging across species and experiments, the operating $c=20 \%$ was reasonably confirmed and appears independent of properties of the sieve tube geometry or the loading mechanism (passive versus active) in plants. The scatter in reported values of $c$ around $c=20 \%$, however, was substantial (Jensen et al. 2013) with many species operating at $c<20 \%$. This low loaded sugar concentration value has also been used to argue against the validity of the Münch mechanism, especially in tall trees (Knoblauch \& Oparka 2012), since it leads to a decrease in mass flux as predicted by the simplified physics. Therefore, it remains open whether plant sugar transport actually operates sub-optimally, or whether alternatives or modifications to the Münch mechanism are necessary to explain long-distance yet suboptimal sugar transport.

Motivated by these issues, we ask to what degree refinements and addressing the model simplifications in the description of the transport physics within the Münch mechanism enhance $J$ above and beyond expectations from earlier theories. To address this question generically, an idealized, unsteady, two-dimensional, osmotically driven pipe flow governed by the physics of the Münch hypothesis is considered. No attempt is made to represent all the complexities of the geometry of the phloem tissue or in the loading and 
unloading mechanisms of sugars. Instead, the main novelty here stems from the inclusion of the simultaneous effects of concentration-dependent viscosity where local changes in viscosity with $c$ (axially and radially) are allowed. It is shown that including such adjustments to viscous stresses lead to significant enhancement in the magnitude of the mass flux, especially in long tubes, when compared to prior one-dimensional (axial) models (Thompson \& Holbrook 2003, Jensen et al. 2016) and globally averaged Poiseuille models (Jensen et al. 2013). Moreover, this enhancement in $J$ is shown to be accompanied by a reduced pressure gradient driving the flow. Thus, the work here adds support to the Münch hypothesis by offering a new perspective regarding the contribution of coordination between axial and radial flow to $J$.

Before describing the new representation of viscous stresses within the context of the Münch hypothesis, some comments and clarifications about efficient sugar transport and its relation to $c$ are illustrated through the occurrence of a maximum sugar flux $J=$ $J_{\max }$ at a well-defined $c$ in globally averaged Poiseuille models. That measured sugar concentration in the leaves of tall trees is lower than this $c$ corresponding to maximum sugar flux was the basis for some critique of the Münch hypothesis (Knoblauch et al. 2016). It is to be noted that the $c$ corresponding to $J_{\max }$ in globally averaged Poiseuille models is shown not to be sensitive to the phloem hydraulic properties or even tube geometry. Hence, the occurrence of such a $c$ is weakly connected to phloem hydraulics as later discussed.

In prior work (Jensen et al. 2013), the sugar mass flux $J\left(\mathrm{~kg} \mathrm{~s}^{-1}\right)$ was assumed to be only advective and given by

$$
J(c)=Q(c) \rho(c) c
$$

where $Q(c)$ is the volumetric flow rate $\left(\mathrm{m}^{3} \mathrm{~s}^{-1}\right), \rho(c)$ is the density of the phloem sap that varies with $c$, and $c$ is the sucrose concentration inside the sieve tube as before. In this approach, the driving force for $Q$ and the constraints on it are now formulated to be $c$ dependent. The maximal flux $J_{\max }$ emerges when solving for $c$ at the critical point $\partial J / \partial c=0$. For $c>0$, the existence of this single critical point is virtually guaranteed provided the water flux $\rho(c) Q(c)$ non-linearly decreases with increasing $c$. For laminar flows in tubes, the Hagen-Poiseuille (HP) equation for $Q$ and the resulting $J$ can be expressed as (Jensen et al. 2013)

$$
Q=X_{f}\left[\frac{\Delta P}{\mu(c)}\right] ; J=X_{f}\left[\frac{\Delta P}{\nu(c)}\right] c ; \nu(c)=\frac{\mu(c)}{\rho(c)},
$$




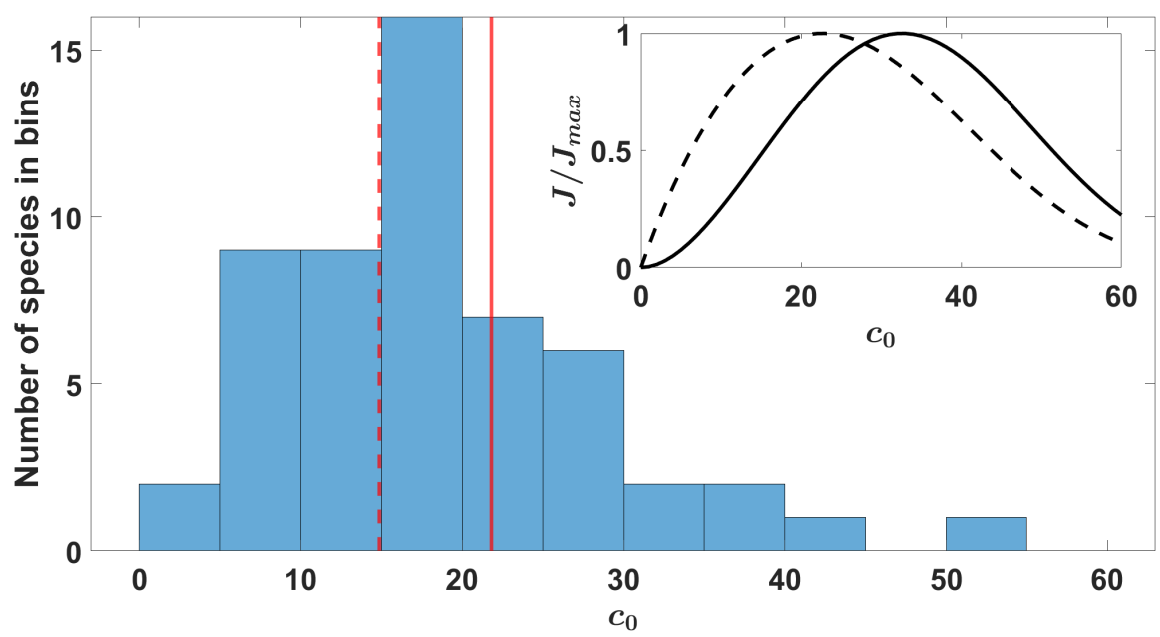

Figure 1: Histogram showing the reported number of species (ordinate) operating at the measured phloem sugar concentration $\left(c_{o}\right)$ values (abscissa) taken from (Jensen et al. 2013). Solid red line $\left(c_{0} \sim 21.8 \%\right)$ denotes the average concentration for active loading species and dashed red line $\left(c_{0} \sim 14.8 \%\right)$ denotes the average concentration for passive loading species. Inset figure shows the computed normalized flux $J / J_{\max }$ for the globally averaged Poiseuille models where the solid black line denotes a concentration dependant pressure gradient (through the osmotic relation) and black dashed line denotes an externally imposed constant pressure gradient.

where $X_{f}$ is a geometric factor that varies with $L$ and $a, \Delta P$ is the pressure difference inside the tube that drives the flow (and need not be only osmotic), $\mu(c)$ is the concentrationdependent dynamic fluid viscosity that increases exponentially with $c$ (Bouchard \& Granjean 1995), and $\nu(c)$ is the kinematic viscosity that also increases with $c$. With increasing $c$, the rise in $\mu(c)$ far exceeds any increase in $\rho(c)$ so that the functional relations of $\mu(c)$ and $\nu(c)$ with $c$ are assumed to be the same (within a constant $\rho$ ). For an order of magnitude illustration, increasing $c$ from $10 \%$ to $50 \%$ increases $\rho$ by a factor of 1.2 whereas $\mu(c)$ increases by a factor of 4 . Because $\nu(c)$ increases non-linearly with $c$ as discussed before, a maximum $J=J_{\max }$ must exist at a corresponding optimal $c$ value that is independent of $X_{f}$. Moreover, the existence of this maximum is not predicated based on the precise details of the osmotic controls on $\Delta P$. Returning to $J_{m a x}$, for a preset $X_{f}$, the hydraulic conductance of the tube $K_{t}$ can be related to the inverse of viscosity using $K_{t}=X_{f} / \mu(c)$. Independent of whether osmotic effects on $\Delta P$ are fully represented, 
a $J_{\max }$ associated with an optimal $c$ can be derived (numerically here) and graphically shown in figure 1 , and it can vary significantly depending on the model presentation within the range of observed values (Jensen et al. 2013).

The Van't Hopf relation approximating the $\Delta P$ solely from osmotic potential (solid line in figure 1 inset) is given by

$$
\Delta P \propto \frac{R_{g} T \rho(c)}{M_{s}} c
$$

where $R_{g}$ is the gas constant, $T$ is the absolute temperature and $M_{s}$ is the molar mass of sugar. Similar results with a single $J_{\max }$ but at lower optimum loading sugar concentration (dashed line in figure 1 inset) are produced using an externally supplied constant pressure difference that varies from 1 to $2 \mathrm{MPa}$ with no concentration dependency. This analysis demonstrates that the existence of a $J_{\max }$ is not tightly connected with the Münch hypothesis in the following sense: the precise functional dependence of $\Delta P$ on $c$ is not necessary for the existence of a $J_{\max }$. Hence, the fact that a $J_{\max }$ exists for a certain $c$ is not particularly informative about how axial-radial coordination in the phloem operates and what the role of radial viscosity in this coordination is.

\section{Results}

This section discusses the effect of including viscosity variations in the flow equations on mass flux $J$. First, a comparison between the constant viscosity model in 2-D (called the Poiseuille model hereafter) and the globally averaged Poiseuille model used to generate the results in figure 1 is presented. This comparison shows the effect of problem set-up and dispersion effects (molecular and Taylor dispersion), which are included in the 2-D Poiseuille (and the 2-D model with variable viscosity) model. Second, the effect of viscosity is discussed by comparing the 2-D model with variable viscosity (called generalized model heareafter) and the Poiseuille model in 2-D (i.e. the two end-member cases discussed is section 4.1). Finally, the enhancement of mass transport due to local coordination between axial and radial movement is presented using the generalized model simulations.

\subsection{Effect of dispersion and problem set-up on $J$ variations with $c_{o}$}

A comparison between the globally averaged Poiseuille model (Jensen et al. 2013) and the Poiseuille model in 2-D that excludes local viscosity effects by using a constant 


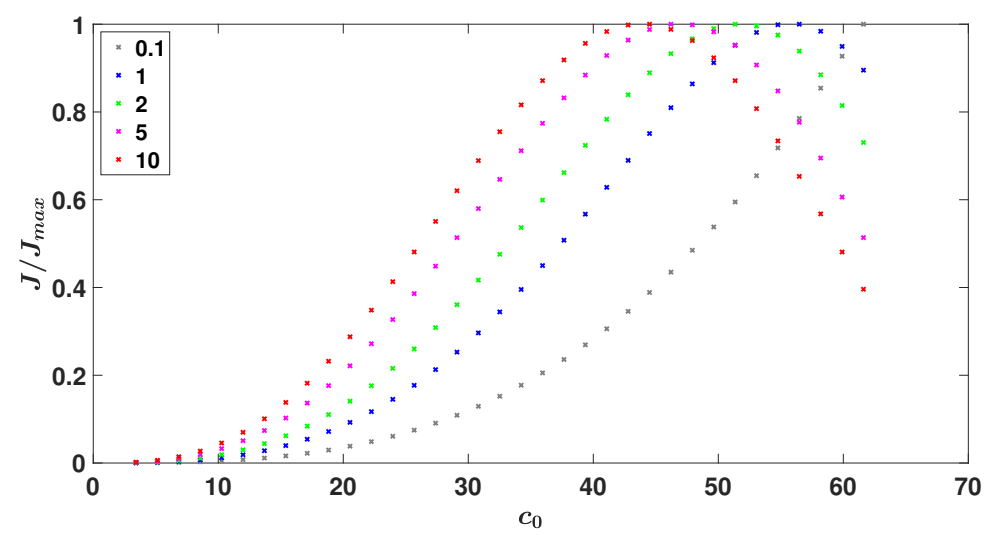

Figure 2: Normalized flux $J / J_{\max }$ as a function of the initial concentration $c_{0}$ for the Poiseuille model in 2-D in a closed tube. The five-tube lengths ( $L$ in $\mathrm{m}$ ) are shown using different colors.

viscosity that depends on $c_{0}$ (Jensen et al. 2016) while keeping molecular diffusion is presented in figure 2 . The relation between $J / J_{\max }$ and $c_{0}$ appears to be similar in shape to the one obtained from the globally averaged Poiseuille model shown in figure 1. Despite the qualitative agreement, three differences are observed. First, the optimal sugar concentration at which $J / J_{\max }=1$ is larger for the Poiseuille model than the globally averaged Poiseuille model for any conduit length (shown in figure 1). Second, there is an increase in the $J / J_{\max }$ versus $c_{0}$ curvature with increasing $c_{0}$ at low $c_{0}$ (i.e. slow rise in the normalized flux for low initial concentrations), and third, the optimal point $J / J_{\max }=$ 1 is different for different tube lengths in each case with short tubes reaching $J / J_{\max }=$ 1 at higher $c_{0}$ than long tubes (figure 2). Recall that the optimal point for the globally averaged Poiseuille model was not affected by the tube length $L$ (not shown). The first two differences between the models are due to the problem set-up where the closed tube assumption requires higher $c_{0}$ to drive the flow as expected. Optimal point difference is attributed to dispersion and molecular diffusion not explicitly resolved in the simplified analysis.

\subsection{Effect of a concentration-dependent viscosity on $J\left(c_{0}\right)$}

Typical phloem conditions are used to generate the results in both models: $a=$ $10 \mu \mathrm{m}, k=5 \times 10^{-14} \mathrm{msPa}^{-1}$ and $D=4 \times 10^{-10} \mathrm{~m}^{2} \mathrm{~s}^{-1}$. The tube length $L$ was varied from 0.1 to $10 \mathrm{~m}$ to describe small and large plants or trees, respectively. The initial sugar 
concentration inside the tube $c_{0}$ was varied from 100 to $1800 \mathrm{~mol} \mathrm{~m}{ }^{-3}$, which does not significantly affect the applicability of the Van't Hopf relation and the Newtonian fluid approximation (to detect the largest signature of viscosity effects on $J / J_{\max }$ see supplemental materials and methods S3 for results on larger variation in $c_{0}$ (up to $2450 \mathrm{~mol}$ $\mathrm{m}^{3}$ ) where the viscosity effects may be overestimated). In this range of values, the normalized flux for the generalized model (not shown) exhibits a similar behavior for the $L=0.1 \mathrm{~m}$ as the Poiseuille case here. However, with increasing $L$, the optimal point where $J / J_{\max }=1$ was not reached for the range of $c_{0}$ studied (not shown). Interestingly, the normalized flux over a wider range of $c_{0}$ for $L=2 \mathrm{~m}$ (selected for illustration) shows that an optimal point does exist for the generalized model as well but $c_{0}$ must operate well outside the range of the Van't Hopf approximation (see supplemental figure $\mathrm{S} 1 \mathrm{~b})$. The fact that $J / J_{\max }=1$ was not attained in the generalized model for the range of $c_{0}$ selected here may lead to the erroneous conclusion that the inclusion of local viscosity changes retards sugar transport. The normalization by $J_{\max }$ hides some facts about the magnitude of $J$, which is much higher for the generalized model for the same $c_{0}$.

Resolving the local changes in viscosity results in an increase in the overall conductivity of the tube above and beyond the 2-D Poiseuille model (figure 3a). The generalized model appears to have far higher $J$ than the Poiseuille model at a given $c_{0}$ for all tube lengths except for $L=0.1 \mathrm{~m}$ where the two models are almost indistinguishable. The effect of the tube length is present in both models where an increase in $L$ leads to an increase in the flux $J$ until a certain value is reached after which $J$ starts to decrease with increasing $L$ (for example, in the generalized model, $J$ when $L=10 \mathrm{~m}$ is lower than $J$ when $L=5 \mathrm{~m}$ for the same $c_{0}$ as discussed next). An interesting observation is that the value of $L$ for which there is a loss of conductivity (the sugar flux decreased for the same initial concentration $c_{0}$ ) is different for both models, $L=5 \mathrm{~m}$ for the generalized model and $L=2 \mathrm{~m}$ for the Poiseuille model. The importance of variable viscosity can be evaluated by the relative difference in sucrose fluxes

$$
e=\left|\frac{J_{g}-J_{p}}{J_{g}+J_{p}}\right|,
$$

where the subscripts 'g' and 'p' denote generalized and Poiseuille, respectively (figure $3 \mathrm{~b})$. The results show that $e$ increases with $L$ and $c_{0}$ as expected. This is because the $c_{0}$ affects the overall viscosity value itself and $L$ affects the development of the velocity profile over which viscosity gradients are allowed to buildup and increase with increasing $L$. The increase in actual mass flux magnitude due to the inclusion of a variable vis- 


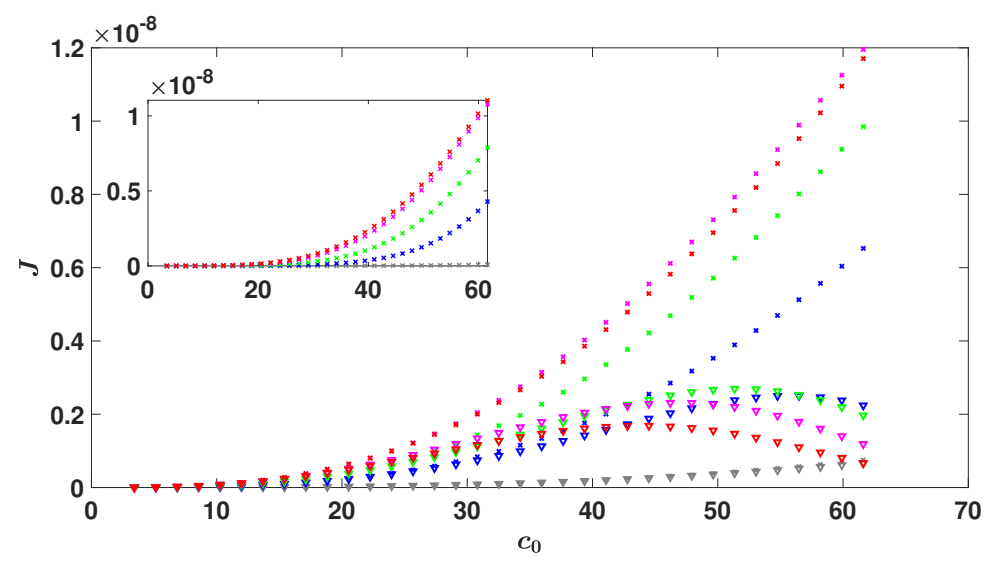

(a)

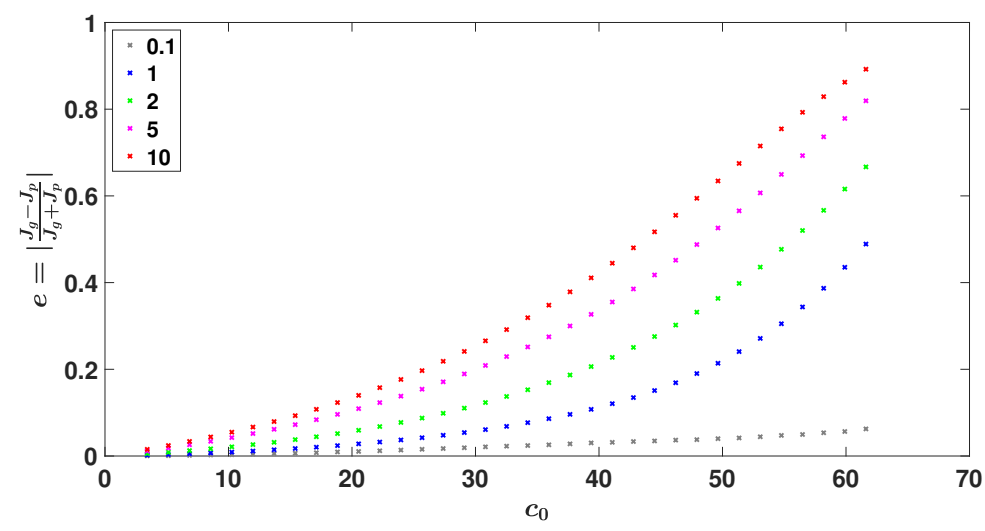

(b)

Figure 3: (a) Sugar mass flux $J\left(\mathrm{Kg} \mathrm{s}^{-1}\right)$ as a function of the initial phloem sugar concentration $c_{0}$ for the generalized model (cross markers) and the Poiseuille model (triangle markers). Inset plot shows the flux for the viscosity effect (difference between both models). (b) Relative difference in sucrose fluxes $e=\left|J_{g}-J_{p}\right| /\left(J_{g}+J_{p}\right)$ as a function of the initial concentration $c_{0}$. Different tube lengths are presented using different colors.

cosity can be approximated by subtracting the flux of the Poiseuille model from the generalized model (Fig 3a inset). As expected, this effect increases with increasing $L$.

\subsection{Two-dimensional flow results}

To understand why the increase in mass flux occurs for the generalized model (visa-vis the Poiseuille), the 2-D simulations are used to illustrate the radial-axial flow and 
their coordination. These simulations show the local effect of concentration gradients on flow velocity components affected by viscosity and its gradients. Model results show that the computed axial and radial velocities are higher in magnitude because of a lower viscosity near the conduit walls (figures $4 \mathrm{a}, 4 \mathrm{~b}, 4 \mathrm{c}$ ). Additionally, the pressure gradient driving the flow is lower compared to the constant viscosity case (Fig $4 \mathrm{~d}$ ). The results presented here are for initial concentration $c_{0}=800 \mathrm{~mol} \mathrm{~m}^{-3}$ and $L=2 \mathrm{~m}$ for the time when the sugar front is located at about $30 \%$ of the conduit length, chosen for illustration only. The time $\tau$ it took for the front to reach this location was different for the models: $\tau=170.5$ for the generalized model and $\tau=204.2$ for the Poiseuille model (i.e. in the Poiseuille model the flow is about 1.2 times slower). Despite this difference in flow velocity, the sugar front at this location appears similar in the models (4a). The highest difference is near the front location. After this position, the difference appears to be enlarged partly due to concentrations being near zero after the fronts. The velocity profile, on the other hand, appears to be wider, and the difference between the models is higher after the front position (Fig 4b). The axial velocity is also higher in the generalized model compared to the Poiseuille model.

The effect of local viscosity gradients generated by concentration gradients is more apparent in the radial velocity than the axial velocity as expected (figure 4c). This finding can be anticipated from equation (11) since the radial velocity profile is directly related to viscosity gradients in the axial direction. These gradients are generated based on concentration gradients from the axial direction that are large, due to the wave nature of the problem (because the advection-diffusion equation has a wave shape especially when it is advection-dominated). The relative difference $e$ between the models is high near the front location. Moreover, the location of the maximum radial velocity is shifted further away from the membrane for the generalized model since the tube conductance has a new term that depends on non-local changes in the radial direction (i.e. $\left\langle K_{t}\right\rangle_{r}$ in equation (10)). Similar to the axial velocity profile, this non-local effect is also apparent in the radial velocity profile for the generalized model that has a wider velocity range, when compared to the Poiseuille model. Moreover, due to a lower sugar concentration near the membrane, the viscosity of the sap decreases leading to less resistance to the radial inflow of water (that is the driving force for osmotically driven flows) in the generalized model. This can be conceptually understood as a decrease in wall-friction when area-averaging the equations. 


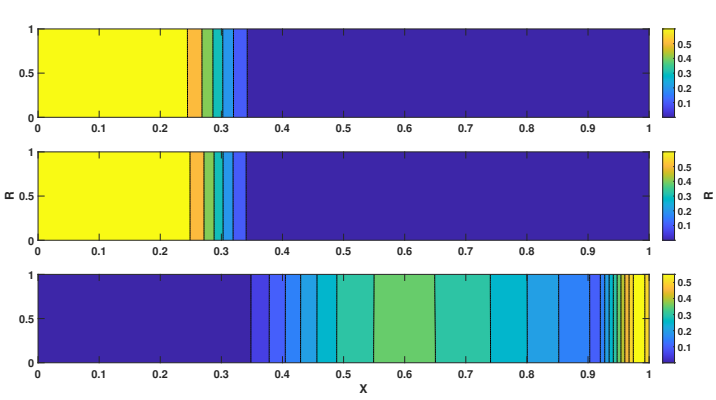

(a)

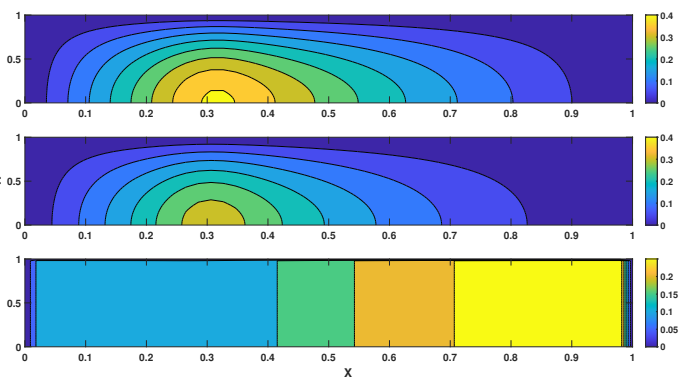

$(b)$

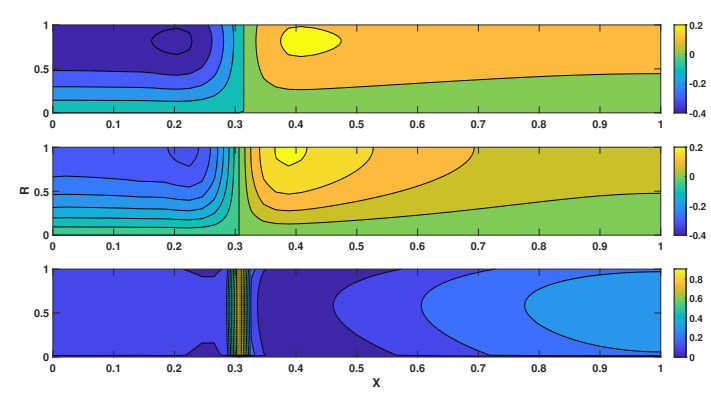

(c)
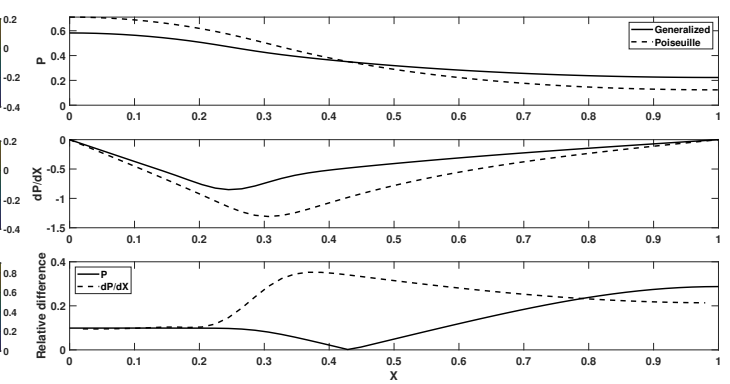

$(d)$

Figure 4: Results for a tube length $L=2 \mathrm{~m}$ and sugar concentration $c_{0}=800 \mathrm{~mol} \mathrm{~m}^{-3}$ when the sugar front is located at approximately $30 \%$ of the domain. Due to cylindrical symmetry, only half of the domain in the radial direction $R$ is shown. (a) concentration profiles, $(b)$ axial velocity profiles, and $(c)$ radial velocity profiles for the generalized model (top), Poiseuille model (middle), and the relative difference (bottom), respectively. $(d)$ pressure (top figure), pressure gradient (middle figure), and their relative difference (bottom figure) for the generalized and Poiseuille models. The relative difference between the models is calculated by $e_{c}=\left|\frac{C_{g}-C_{p}}{C_{g}+C_{p}}\right|$ where the subscripts ' $\mathrm{g}$ ' and 'p' denote generalized and Poiseuille models, respectively.

The generalized model also has a smoother pressure field compared to the Poisseuille model (figure 4d). An interesting result in this figure is that the global pressure gradient over the domain is smaller for the generalized model and yet the front travels at a higher speed. This paradoxical result can only be explained by the increase in conductivity of the tube because of local viscosity effects being coordinated in radial and axial directions. 


\section{Discussion and concluding remarks}

The concentration-dependent viscosity has two effects on $J$ - both leading to an increase in its magnitude. The first is that the smaller viscosity in the vicinity of the membrane wall ( $c$ at the membrane is set by clear water in the xylem) results in an increase in $v$ into the pipe wall region. This increase has the effect of radially advecting sugar molecules away from the pipe walls and towards the pipe center. Because the axial velocity profile peaks in the pipe center yet the radial viscous stress is zero there, the overall mass flux is also increased. The second is that the increase in radial velocity near the pipe walls, when coupled to a zero radial velocity at the pipe center (as required by symmetry), must be accompanied by an increase in axial velocity gradients due to the incompressibility approximation. Thus, a speeding up of $u$ is expected. The analysis of the axial pressure distribution further suggests that this effect is sensed over a broader region of $L$. This speeding up yields a faster front advancement of sugar away from the loading zone. Both mechanisms are operating in concert to increase $J$ above and beyond Poiseuille's model. That those two effects act together to enhance tube permeability affecting $J$, not the driving force for water (i.e. pressure gradients) is also supported by the analysis here. For this reason, the $J_{\max }$ analysis and its dependence on $c_{o}$ leading to $c_{o} \approx 20 \%$ in Figure 1 being not sensitive to $X_{f}$ (as earlier shown) also cannot detect this coordination between axial and radial flow.

The importance of this finding is also highlighted when comparing typical concentration values for crops (that are mainly active sugar loaders) and trees (mainly passive loaders). From figure 1, active loaders have a higher concentration than passive loaders. For these high concentrations (for example, maize has $c_{0} \sim 50 \%$ ), the simplified Poiseuille model predicts an optimal length around $2 \mathrm{~m}$ where the generalized model predicts an optimal length around $5 \mathrm{~m}$ (shown in figure 3a). For low concentrations (for example a pine tree), the generalized model shows more resilience for increasing phloem length when compared to the Poiseuille model (shown in figure $3 \mathrm{~b}$ ).

To summarize, the effect of a concentration-dependent viscosity on sucrose transport in phloem allowed the flow to have a higher velocity for the same initial concentration, especially for long tubes. In addition, it resulted in a lower pressure gradient driving the flow along the axial direction. This finding contributes to the growing evidence that the pressure-flow hypothesis can provide the necessary mechanism for long-distance 
sugar transport as long as the complexity in transport physics is accommodated. It is to be emphasized that the work here showed that viscosity adjustments lead to conductivity enhancement for sugar transport instead of pressure gradients for water flow.

More negative xylem water tension requires a higher osmotic potential to maintain phloem transport in dry conditions (Sevanto 2014). During drought, the increase in optimal sugar concentration operating range for $J$ would allow plants to increase their sucrose concentration to potentially overcome those large tensions arising in the xylem without a substantial decrease in flow rate. Future work will focus on the effects of nonlinear xylem water potential on phloem transport by including sugar sources and sinks along the phloem path.

\section{Materials and methods}

To isolate the effect of a concentration-dependent viscosity on radial-axial flow coordination, many simplifications must still be invoked when representing the physics of translocation in a cylindrical tube. In all formulations considered here, it is assumed that i) the phloem vasculature can be approximated by a long slender tube of length $L$ and radius $a(\epsilon=a / L<<1)$ with rigid semipermeable walls characterized by a constant permeability $k$ that allows the exchange of water molecules but not sugars with the surroundings, ii) sieve plates have minimal effect on the flow and can be modeled as either an 'extra' drag force uniformly acting along with $L$ or ignored altogether, (iii) the bulk flow is at very low Reynolds number $R e \sim \rho a u \mu^{-1} \ll 1$ where $u$ is a characteristic longitudinal velocity, $\mu$ the dynamic viscosity, and $\rho$ the density, so that creeping flow is maintained throughout, iv) sugar sources and sinks are modeled as boundary conditions at the entry and exit end of the tube. Hence, water can be exchanged with the surroundings but not sugars thereby suppressing any enhancement due to a relay effect.

\subsection{Variable Viscosity Model}

To derive the general model that includes concentration and viscosity variations in axial and radial directions, the governing equations under certain assumptions and simplifications are first analyzed in Cartesian coordinates. Then, the non-dimensional form of these equations, which are necessary for analyzing the numerical model results, are presented in cylindrical coordinates. The model to be presented in this section is con- 
sidered one 'end-member' case in including a concentration-dependent viscosity. The other 'end-member' case this model is compared to is the Poiseuille model that assumes a constant viscosity in the domain set by the initial loading concentration. An example of this model in a globally-averaged case is the model discussed by Jensen et al. (2013). Thompson \& Holbrook (2003) present a different model that is between these 'end-member' cases. In their work, they included local variations in viscosity inside the domain but only using radially averaged equations (i.e. variations in viscosity along radial directions ignored). Due to the nonlinear relationship in the viscous stress between velocity and viscosity, areaaveraging the equations leads to a simplified model that excludes the effect of this nonlinearity. This issue can be resolved at the expense of solving the equations in axial and radial directions and frames the main approach here.

\subsubsection{Governing equations}

In a three-dimensional Cartesian coordinate system defined by longitudinal $\left(x_{1}=\right.$ $x)$, lateral $\left(x_{2}=y\right)$, and vertical $\left(x_{3}=z\right)$ directions, water flow within the phloem satisfies the continuity equation

$$
\frac{\partial \rho}{\partial t}+\frac{\partial\left(\rho u_{i}\right)}{\partial x_{i}}=0
$$

where $t$ is time, $i=1,2,3$ describe direction $x_{i}$, and $u_{i}$ is the instantaneous velocity along direction $x_{i}$. Here, index notation is used with repeated indices implying summation unless otherwise stated. The flow must also satisfy the conservation of momentum, which describes the force balance along direction $x_{i}$, and is given as

$$
\frac{D\left(\rho u_{i}\right)}{D t}=\frac{\partial}{\partial x_{j}} \sigma_{i j}
$$

where $D(.) / D t$ is the material derivative and $\sigma_{i j}$ are the nine components of the stress tensor. The $\sigma_{i j}$ of a Newtonian fluid can be approximated by

$$
\sigma_{i j}=-p \delta_{i j}+\mu\left(\frac{\partial u_{j}}{\partial x_{i}}+\frac{\partial u_{i}}{\partial x_{j}}\right)
$$

where $p$ and $\mu$ are the local pressure and dynamic viscosity of the fluid, respectively, and the $\delta_{i j}$ is the Kronecker delta (i.e. $\delta_{i j}=0$ when $i \neq j$ but unity otherwise). This representation of $\sigma_{i j}$ is approximate and assumes that the stress tensor is symmetric $\left(\sigma_{i j}=\right.$ $\left.\sigma_{j i}\right)$ and the so-called second viscosity coefficient (or volume viscosity) is momentarily ignored (Panton 2006). 
In terms of fluid properties, $\rho$ depends on $c$ and strictly speaking cannot be treated as constant when $c$ varies in time or along $x_{i}$. However, this dependence is minor when compared to variations in $\mu$ as demonstrated earlier (see section 1) and variations in $\rho$ will be assumed small for simplicity so that $\partial u_{i} / \partial x_{i}=0$. In this case, the $\sigma_{i j}$ representation given by equation (7) is reasonable (Panton 2006). Another common assumption in phloem transport is that $\mu$ is constant set by the loading concentration. This approximation is only applicable for small concentration values. However, in plants, $c$ can range from $15 \%$ to $35 \%$ (and for maple trees even up to $\approx 50 \%$ ). In this high concentration range, the dependence of viscosity on concentration has not been fully analyzed in the context of three-dimensional water and sugar transport. Some models include this dependence of $\mu$ on $c$ in an area-averaged formulation (Thompson \& Holbrook 2003, Jensen et al. 2016), but area-averaged formulations that evolve concentration axially and presume uniform concentration along the radial direction cannot resolve radial-axial flow coordination to be studied here. Therefore, the model proposed here includes the dependence of $\mu$ on $c$ in both axial and radial directions and tracks its consequences on the shape of the $J-c$ relation as well as the magnitude of $J$ across differing $L$ and loading concentrations.

In terms of flow properties, the low Reynolds number $(R e \ll 1)$ and small aspect ratio $\left(\epsilon \approx 10^{-5}\right)$ can be used to show that under the so-called lubrication theory (where the flow in one of the dimensions is significantly smaller than the others because of geometric constraints), the momentum balance may be approximated by (now written in cylindrical coordinates)

$$
\mu \frac{\partial u}{\partial r}=\frac{r}{2} \frac{\partial p}{\partial x}, \quad \frac{\partial p}{\partial r}=0
$$

where $x$ remains the axial direction with $x=0$ situated at the loading zone, $r$ is the radial direction with $r=0$ defining the center of the tube, and $u(x, r)$ and $v(x, r)$ are the axial and radial velocity components respectively at any point $(x, r)$. In supplemental materials and methods S1, the derivation of this formulation and all its assumptions are presented for completeness.

To understand how viscosity gradients affect the flow, equation (8) can be written in a compact form. First, the tube conductance is re-defined as $K_{t}=1 / \mu$ as in section 1 (the geometric factor $X_{f}$ is absent since it is the result of radial averaging). Integrating equation (8) in the radial direction while noting that the pressure $p$ is only a func- 
tion of the axial position (as shown in supplemental materials and methods S1) leads to

$$
u(x, r)=\frac{1}{2} \frac{\partial p}{\partial x} \int_{0}^{r} r K_{t}(x, r) \mathrm{d} r+A(x),
$$

where $A(x)$ is an integration function that varies in $x$. Using the no-slip boundary condition on the longitudinal velocity component $u(x, a)=0$ at the membrane surface set as $r=a$ leads to $A(x)=-\left(a^{2}\left\langle K_{t}\right\rangle / 4\right)(\partial p / \partial x)$ where $\left\langle K_{t}\right\rangle$ is the radially averaged tube conductance. Similarly, the term $\int_{0}^{r} K_{t} \mathrm{~d} r$ can be written as $r^{2}\left\langle K_{t}\right\rangle_{r} / 2$ where the subscript $r$ denotes radial averaging until the current radial position $(r \leq a)$. Using this form, equation (9) describes the axial velocity

$$
u(x, r)=\frac{1}{4} \frac{\partial p}{\partial x}\left(r^{2}\left\langle K_{t}\right\rangle_{r}-a^{2}\left\langle K_{t}\right\rangle\right),
$$

where both terms $\left\langle K_{t}\right\rangle_{r}$ and $\left\langle K_{t}\right\rangle$ are functions of $x$ but only $\left\langle K_{t}\right\rangle_{r}$ is a function of $r$.

Equation (10) is different from the HP expression because the variable viscosity depends on $c(x, r)$ that itself varies radially and axially. To be clear, this dependence is nonlocal because of the integral operator in the $r$ direction. However, if a constant viscosity is used at a given $x,\left\langle K_{t}\right\rangle_{r}$ and $\left\langle K_{t}\right\rangle$ are equal to $1 / \mu$, and the aforementioned conservation of momentum equation becomes equivalent to the HP expression with an adjustment. This adjustment is due to osmosis that generates a radial inflow of water leading to $\partial^{2} p / \partial x^{2} \neq 0$, which then leads to a variable pressure gradient instead of a constant one as is common in HP applications in pipes (Phillips \& Dungan 1993). However, the partial $\partial p / \partial x$ not being constant does not violate or invalidate the HP equation as discussed elsewhere (Thompson \& Holbrook 2003, Nakad et al. 2021).

The effect of viscosity gradients is not directly apparent in equation (10). To make it explicit, the continuity equation (5) in cylindrical coordinates is now considered. It is given as

$$
\frac{\partial u}{\partial x}+\frac{1}{r} \frac{\partial r v}{\partial r}=0
$$

Here $\rho$ variation with $c$ is once again assumed to be small compared to the viscosity variations with $c$ as stated before. Using the expression for the axial velocity from equation (10) in the continuity equation (11), one can see how axial viscosity gradients impact the radial velocity $v$, which is not identically zero due to osmosis. Moreover, the viscosity gradient is not only the result of the area-averaged tube conductance $\left\langle K_{t}\right\rangle$ but also stems from the radially-averaged (or non-local) tube conductance $\left\langle K_{t}\right\rangle_{r}$ that depends on radial position $r$. Equation (8) with equation (11) can be used to describe the flow of wa- 
ter characterized by $u(x, r)$ (axial velocity) and $v(x, r)$ (radial velocity) inside the tube as a function of position $x, r$.

Equations (8) and (11), however, remain incomplete since there are two equations with three unknowns $u, v$, and $p$. This mathematical setup is in sharp contrast to flow in closed pipes where $v=0$ everywhere due to the solid wall boundary condition at $r=$ $a$ and symmetry considerations at the pipe center. In phloem, osmosis necessitates a finite $v$ at the pipe walls while symmetry considerations alone result in $v=0$ at the center of the pipe. Thus, the third equation that relates $v$ to total pressure inside the tube must be provided by osmoregulation. This equation is best formulated as a boundary condition describing a flow through a porous media (a thin membrane here) at $r=a$. Such a boundary condition may be given by a Darcy-type formulation assuming a very low Reynolds number for the radial flow into or out of the pipe walls. This boundary condition yields an expression for $v$ at $r=a$ given by

$$
\left.v\right|_{r=a}=k\left(p-\left.\Pi\right|_{r=a}\right),
$$

where $\left.\Pi\right|_{r=a}$ is the osmotic potential at the membrane and $k$ is the membrane permeability assumed constant and independent of $v$ (i.e. no Forscheimer or quadratic corrections to Darcy's law). This osmotic potential can be related to $c$ using the Van't Hoff relation, $\Pi=R_{g} T c(x, a)$ as before. This approximation is reasonable for low $c$ and compatible with the assumption that the density and molecular diffusion (discussed below) do not vary appreciably with $c$ when compared to viscosity.

The last equation needed to describe the physics of sugar transport is the conservation of solute mass, which is also needed to solve for $u, v$, and $p$. This equation is derived using Reynolds transport theorem that describes the movement of solutes (mainly sugar here) due to advection and molecular diffusion. In cylindrical coordinates, it is given by

$$
\frac{\partial c}{\partial t}+\frac{\partial}{\partial x}(u c)+\frac{1}{r} \frac{\partial}{\partial r}(r v c)=D \frac{\partial^{2} c}{\partial x^{2}}+D \frac{1}{r} \frac{\partial}{\partial r}\left(r \frac{\partial c}{\partial r}\right),
$$

where $D=\nu S c^{-1}$ is the molecular diffusion coefficient of sugar in water assumed to be again insensitive to $c$ variations when compared to $\nu$, and $S c \gg 1$ is the molecular Schmidt number for sugars in water (usually of order $10^{4}$ ).

The final step for describing the physics of sugar transport is to specify the boundary and initial conditions. These are problem-specific and are selected here to illustrate 
one restrictive 'end-member' case of flow in a closed tube with no sugar sinks. This case is dynamically interesting because sugar concentration keeps building up as no sugars are removed from the tube. The other 'end-member' case is where sugars are instantly consumed at the end of the pipe (i.e $c(L, r)=0$ and sugar sinks are treated as 'infinite'). This latter case is expected to lead to a much larger $J$ in the tube, which is why the focus is on the more restrictive former case. In plants, $c(L) / c(0)<<1$ and thus osmotic gradients are much higher in the presence of sinks than those set by the closed tube assumption. Thus, the physics of closed tubes must require higher loading concentrations to drive the water velocity, which is why they are more restrictive and thus dynamically interesting from the perspective of exploring limitations on the Münch hypothesis. In a pipe closed at both ends $u(x=0)=u(x=L)=0$, water flow must accelerate to a well-defined maximum and then decelerate to zero velocity along $L$. For initial conditions selected here, sugar is released as an axially smooth function $c(x, t=0)=$ $f(x)$ with no radial variation, meaning that radial diffusion is initially fast enough to ensure a uniform distribution of sucrose along $r$. The closed tube assumption with no sinks requires sugar mass to be conserved inside the tube during the entire period resulting in

$$
\begin{gathered}
\left.\frac{\partial c}{\partial x}\right|_{x=0}=\left.\frac{\partial c}{\partial x}\right|_{x=L}=0 \\
\left.(v c)\right|_{r=a}-\left.D \frac{\partial c}{\partial r}\right|_{r=a}=0 .
\end{gathered}
$$

Finally, a no-slip boundary condition at the membrane in the axial direction only, i.e. $\left.u\right|_{r=a}=0$, and symmetry considerations at the center of the tube, where $\left.v\right|_{r=0}=0$ and $\partial c /\left.\partial r\right|_{r=0}=0$, are all enforced.

\subsubsection{Non-dimensional form and key dimensionless numbers}

To elucidate the key dimensionless numbers governing water and sugar movement, and make interpretation of the equations easier, this section describes the scaling analyses and the non-dimensional form of the equations used. To write the equations in dimensionless form, the following scales were adopted: The $x$ and $r$ were scaled by the tube length $L$ and radius $a$, respectively, leading to $x=L X, r=a R$. Time, and axial and radial velocity as well as pressure and concentration were scaled by their respective initial values at $x=0$ (subscript 0 ) leading to $t=t_{0} \tau, u=u_{0} U, v=v_{0} V, p=p_{0} P$, $c=c_{0} C$ and $\mu=\mu_{0} \tilde{\mu}$. The dynamic viscosity $\mu$ was scaled by $\mu_{0}$, determined from $c_{0}$ and $T$. This $\mu_{0}$ will also be used in a constant viscosity model (called the Poiseuille 
model hereafter) as a reference to assess the impact of accommodating variable viscosity in $\sigma_{i j}$ and its spatial gradients. Using these scales, the non-dimensional equations for the two velocity components and pressure are

$$
\begin{gathered}
\frac{\partial U}{\partial X}+\frac{1}{R} \frac{\partial R V}{\partial R}=0 . \\
\tilde{\mu} \frac{\partial U}{\partial R}=\frac{R}{2} \frac{\partial P}{\partial X} \\
\left.V\right|_{R=1}=M P-\left.C\right|_{R=1}
\end{gathered}
$$

where $u_{0}=k R_{g} T c_{0} \epsilon^{-1}, v_{0}=\epsilon u_{0}, p_{0}=\mu_{0} L u_{0} a^{-2}$, and $M=k \mu_{0} L^{2} a^{-3}$ is the Münch number defined as the ratio of axial resistance over radial resistance as discussed in (Jensen et al. 2009, Nakad et al. 2021). The complete scaling analysis for the Navier-Stokes equations is shown in supplemental materials and methods S1.

The non-dimensional form of the conservation of sugar mass, i.e. equation (6), is

$$
\frac{\partial C}{\partial \tau}+P e \frac{\partial}{\partial X}(U C)+P e \frac{1}{R} \frac{\partial}{\partial R}(R V C)=\epsilon^{2} \frac{\partial^{2} C}{\partial X^{2}}+\frac{1}{R} \frac{\partial}{\partial R}\left(R \frac{\partial C}{\partial R}\right)
$$

where $t_{0}=a^{2} D^{-1}$ is the radial diffusion timescale and $P e=v_{0} a D^{-1}$ is the radial Peclet number defined by the ratio of radial advection to radial diffusion. This expression explicitly shows the relative contributions of radial flow dynamics (through $P e$ ) and simplified geometry (through the slender ratio $\epsilon$ ) to mass transport.

\subsection{Model calculations}

The proposed model calculations provide the variations of $J(x, r), c(x, r), u(x, r)$, $v(x, r)$, and $p(x, r)$ at every $t$. A description highlighting the numerical method used to obtain the results is presented in supplemental materials and methods S2. To link a representative $J$ with a $c_{0}$ in a manner that allows comparison with the prior $J_{\max }$ and optimal $c$ analysis, the following steps and approximations were taken in post-processing the model results. With a radial Peclet number $P e \ll 1$, it is reasonable to assume that the mass flow primarily occurs in the axial direction (Nakad et al. 2021). The molecular diffusion can also be neglected since the axial Peclet number, defined by the ratio of axial advection to axial diffusion and derived from $P e$ by $P e_{l}=\epsilon^{-2} P e$, is large (i.e. $\left.P e_{l} \gg 1\right)$. Using these assumptions, the area-averaged sugar flux can be reasonably determined from post-processing time variations of the sugar front position $x_{f}$. This front is also delineated from maximal $|\partial c(x, r) / \partial x|$. To determine $x_{f}$, we fitted an exponen- 
tial relation between $x_{f}$ and $t$ using (Jensen et al. 2009, Nakad et al. 2021)

$$
x_{f}=L-(L-l) \exp \left(\frac{-t}{\hat{t}}\right),
$$

where $l \approx 0.2$ is the initial sucrose front position, $L=1$ is the length of the tube and $x_{f}$ is the front position all in non-dimensional form. Subtracting $l$ from both sides of equation 17, a linear relation between $t$ and $\ln \left[\left(X_{\text {final }}-X_{f}\right) / X_{\text {final }}\right]$ (where $X_{\text {final }}=L-$ $l \approx 0.8$ and $X_{f}=x_{f}-l$ ) can be obtained. Hence, linear regression applied to the $2 \mathrm{D}$ numerical solution was then used to obtain the constant $\hat{t}$ away from the entrance boundary condition. The front speed was determined as $U_{s}=d x_{f} / d t$. Finally, the mass flux was approximated by $J_{\text {num }}=Q_{\text {num }} C$ where $Q_{\text {num }}$ is the numerical volumetric flow rate. The $Q_{\text {num }}$ was determined using approximated speed $Q_{\text {num }}=A_{t} U_{s}$ where $A_{t}$ is the cross-sectional area of the tube. We present results from the two-dimensional (2-D) model simulation for the axial velocity $U$, radial velocity $V$, concentration $C$, and pressure $P$ in the dimensionless form to illustrate the effect of variable viscosity on radial and axial variations of these variables. The 2 -D model with variable viscosity is hereafter referred to as the generalized model. The model simulations were conducted using MATLAB programming language (Mathworks, Natick, MA).

\section{Acknowledgments}

MN, J-CD, and GK acknowledge support from the U.S. National Science Foundation (NSFAGS-1644382, NSF-IOS-1754893 and NSF-AGS-2028633). SS was supported by Los Alamos Directed Research and Development Exploratory Research Grant (No. 20160373ER). No data are generated or used in this work.

\section{References}

Bouchard, C. \& Granjean, B. P. (1995), 'A neural network correlation for the variation of viscosity of sucrose aqueous solutions with temperature and concentration', LWT-Food Science and Technology 28(1), 157-159.

Curtis, O. \& Scofield, H. (1933), 'A comparison of osmotic concentrations of supplying and receiving tissues and its bearing on the Münch hypothesis of the translocation mechanism', American Journal of Botany pp. 502-512.

Fatichi, S., Pappas, C., Zscheischler, J. \& Leuzinger, S. (2019), 'Modelling carbon sources and sinks in terrestrial vegetation', New Phytologist 221(2), 652-668. 
Fensom, D. (1981), 'Problems arising from a Münch-type pressure flow mechanism of sugar transport in phloem', Canadian Journal of Botany 59(4), 425-432.

Hölttä, T., Mencuccini, M. \& Nikinmaa, E. (2009), 'Linking phloem function to structure: analysis with a coupled xylem-phloem transport model', Journal of Theoretical Biology 259(2), 325-337.

Housley, T. \& Fisher, D. (1977), 'Estimation of osmotic gradients in soybean sieve tubes by quantitative autoradiography: qualified support for the Münch hypothesis', Plant Physiology 59(4), 701-706.

Huang, C., Domec, J., Palmroth, S., Pockman, W., Litvak, M. \& Katul, G. (2018), 'Transport in a coordinated soil-root-xylem-phloem leaf system', Advances in Water Resources 119, 1-16.

Jensen, K., Berg-Sørensen, K., Bruus, H., Holbrook, N., Liesche, J., Schulz, A., Zwieniecki, M. A. \& Bohr, T. (2016), 'Sap flow and sugar transport in plants', Reviews of Modern Physics 88(3), 035007.

Jensen, K., Berg-Sørensen, K., Friis, S. M. \& Bohr, T. (2012), 'Analytic solutions and universal properties of sugar loading models in Münch phloem flow', Journal of Theoretical Biology 304, 286-296.

Jensen, K. H., Savage, J. A. \& Holbrook, N. M. (2013), 'Optimal concentration for sugar transport in plants', Journal of the Royal Society Interface 10(83), 20130055.

Jensen, K., Rio, E., Hansen, R., Clanet, C. \& Bohr, T. (2009), 'Osmotically driven pipe flows and their relation to sugar transport in plants', Journal of Fluid Mechanics 636, 371-396.

Knoblauch, M., Knoblauch, J., Mullendore, D., Savage, J., Babst, B., Beecher, S., Dodgen, A., Jensen, K. \& Holbrook, N. (2016), 'Testing the Münch hypothesis of long distance phloem transport in plants', Elife 5, e15341.

Knoblauch, M. \& Oparka, K. (2012), 'The structure of the phloem: still more questions than answers', The Plant Journal 70(1), 147-156.

Knoblauch, M. \& Peters, W. (2017), 'What actually is the Münch hypothesis? a short history of assimilate transport by mass flow', Journal of Integrative Plant Biology 59(5), 292-310.

Konrad, W., Katul, G., Roth-Nebelsick, A. \& Jensen, K. (2018), 'Xylem functioning, dysfunction and repair: a physical perspective and implications for phloem 
transport', Tree Physiology 39(2), 243-261.

Lang, A. (1979), 'A relay mechanism for phloem translocation', Annals of Botany 44(2), 141-145.

Liesche, J. \& Schulz, A. (2018), 'Phloem transport in gymnosperms: a question of pressure and resistance', Current Opinion in Plant Biology 43, 36-42.

Mencuccini, M. \& Hölttä, T. (2010), 'The significance of phloem transport for the speed with which canopy photosynthesis and belowground respiration are linked', New Phytologist 185(1), 189-203.

Münch, E. (1930), Stoffbewegungen in der Pflanze, G. Fischer, Jena, Germany.

Nakad, M., Witelski, T., Domec, J., Sevanto, S. \& Katul, G. (2021), 'Taylor dispersion in osmotically driven laminar flows in phloem', Journal of Fluid Mechanics 913.

Nikinmaa, E., Hölttä, T., Hari, P., Kolari, P., Mäkelä, A., Sevanto, S. \& Vesala, T. (2013), 'Assimilate transport in phloem sets conditions for leaf gas exchange', Plant, Cell \& Environment 36(3), 655-669.

Panton, R. L. (2006), Incompressible flow, John Wiley \& Sons.

Phillips, R. \& Dungan, S. (1993), 'Asymptotic analysis of flow in sieve tubes with semi-permeable walls', Journal of Theoretical Biology 162(4), 465-485.

Salmon, Y., Dietrich, L., Sevanto, S., Hölttä, T., Dannoura, M. \& Epron, D. (2019), 'Drought impacts on tree phloem: from cell-level responses to ecological significance', Tree Physiology 39(2), 173-191.

Savage, J., Beecher, S., Clerx, L., Gersony, J., Knoblauch, J., Losada, J., Jensen, K., Knoblauch, M. \& Holbrook, N. (2017), 'Maintenance of carbohydrate transport in tall trees', Nature Plants 3(12), 965.

Sevanto, S. (2014), 'Phloem transport and drought', Journal of experimental botany 65(7), 1751-1759.

Sevanto, S. (2018), 'Drought impacts on phloem transport', Current Opinion in Plant Biology 43, 76-81.

Sevanto, S., Mcdowell, N. G., Dickman, L. T., Pangle, R. \& Pockman, W. T. (2014), 'How do trees die? a test of the hydraulic failure and carbon starvation hypotheses', Plant, cell \&3 environment 37(1), 153-161.

Stanfield, R. C., Hacke, U. G. \& Laur, J. (2017), 'Are phloem sieve tubes leaky conduits supported by numerous aquaporins?', American Journal of Botany 

104(5), 719-732.

Swanson, C. \& Geiger, D. (1967), 'Time course of low temperature inhibition of sucrose translocation in sugar beets', Plant Physiology 42(6), 751-756.

Thompson, M. \& Holbrook, N. (2003), 'Application of a single-solute non-steadystate phloem model to the study of long-distance assimilate transport', Journal of Theoretical Biology 220(4), 419-455.

van Bel, A. (2003), 'The phloem, a miracle of ingenuity', Plant, Cell \&3 Environment 26(1), 125-149.

Wardlaw, I. F. (1968), 'The control and pattern of movement of carbohydrates in plants', The Botanical Review 34(1), 79-105. 